\title{
hMSH6: a potential diagnostic marker for oral carcinoma in situ
}

\author{
Maryam Jessri, ${ }^{1}$ Andrew J Dalley, ${ }^{1}$ Camile S Farah ${ }^{1,2}$
}

${ }^{1}$ UQ Centre for Clinical Research, The University of Queensland, Brisbane, Queensland, Australia ${ }^{2}$ The Australian Centre for Oral Oncology Research \& Education, Brisbane, Queensland, Australia

\section{Correspondence to} Prof Camile S Farah, The Australian Centre for Oral Oncology Research \& Education (ACORE), PO Box 88, Royal Brisbane \& Women's Hospital, Herston, QLD 4029, Australia;

camile@oralmedpath.com.au

Received 6 May 2014 Revised 3 October 2014 Accepted 9 October 2014 Published Online First 28 October 2014

\section{ABSTRACT}

Oral medicine specialists rely upon accurate assessment of pathology to rationalise lesion management, especially for high-risk oral epithelial dysplasia, carcinoma in situ (CIS) and oral squamous cell carcinoma. Cross-discipline cancer research has highlighted the role of genetic instability in neoplasia. Improved diagnostic stringency from translation of immunostaining for DNA repair defects into current pathology practice has potential to benefit pathologists, clinicians and patients. The focus of this study was the obligatory and non-obligatory components of the MutL $\alpha$ and MutS $\alpha$ mismatch repair heterodimers, namely hMLH1, hMSH2, hPMS2 and hMSH6, which were studied in 274 formalin-fixed paraffin-embedded sections. A readily apparent inverse correlation between oral disease severity and both obligatory and nonobligatory components of MutL $\alpha$ and MutS $\alpha$ was observed (hMLH1, $\rho=-0.715$; hPMS2, $\rho=-0.692$; hMSH2, $\rho=-0.728$; and hMSH6, $\rho=-0.702$ ), with particularly conspicuous loss of hMSH6 expression from the stratum basale of $\mathrm{CIS}$.

\section{INTRODUCTION}

Mismatch repair (MMR) pathways encompass the strand-specific, postreplicative DNA repair mechanisms that are key to maintenance of genomic integrity and stability. Numerous MMR proteins intervene to repair DNA biosynthetic errors, singlebase substitution mismatches and insertions/deletions in microsatellites. ${ }^{1}$ The most abundant MMR heterodimer, MutS $\alpha$, initiates the repair mechanism and is composed of hMSH2 and hMSH6. ${ }^{2}$ Subsequently, the MutL $\alpha$ heterodimer is instrumental in excising and correcting mismatched nucleotides and is composed of hMLH1 and hPMS2. ${ }^{3}$

Oral squamous cell carcinoma (OSCC) accounts for $90 \%$ of head and neck cancer which globally represents the sixth highest rate of cancer mortality. ${ }^{4}$ OSCC can be attributed to both environmental and genetic factors. The presence and severity of oral epithelial dysplasia (OED) in oral potentially malignant lesions (OPML) is the most important indication of increased neoplastic transformation risk. ${ }^{4}{ }^{5}$ Impaired expression of MMR genes has been demonstrated for OSCC $;^{67}$ a process that can involve hypermethylation of their promoter regions. ${ }^{8} 9$ Genetic modification of the MMR pathway has also been investigated for OPML, although to a lesser extent. ${ }^{7} 1011$

We hypothesised correlation between MMR protein immunoexpression and the pathology grading of OED in OPML and differentiation in OSCC. Accordingly, we compared MutS $\alpha$ and MutL $\alpha$ expression in pathologist-graded biopsies of
OSCC (well-differentiated or poorly differentiated), OPML (low-risk (LD) or high-risk dysplasia (HD) including carcinoma in situ (CIS)) compared with normal oral mucosa.

\section{MATERIALS AND METHODS \\ Patient samples}

Two hundred and seventy-four archival formalinfixed paraffin-embedded specimens: 113 OSCC, 34 HD including CIS, $37 \mathrm{LD}$ and 90 normal oral mucosa were retrieved and rediagnosed according to WHO criteria by oral pathologist (CSF) (table 1). ${ }^{12}$

\section{Immunohistochemistry}

Five micrometre sections were incubated overnight at $4^{\circ} \mathrm{C}$ with mouse monoclonal antihuman antibodies (Biocare Medical, Concord, California, USA): hMLH1 (G168-15), hPMS2 (A 16-4), hMSH2 (FE11) and hMSH6 (BC/44). Secondary antibody and revealing agent were obtained from Biocare Medical (MACH1 Universal HRP polymer kit, M1U539L10, Biocare Medical, Concord California, USA). Positive controls were from colon, and routine negative staining was performed.

\section{Scoring}

All epithelial layers from 16 randomised microscopic fields $(\times 400)$ were scored. Stain intensity was scaled: $0=$ no stain (equivalent to negative control) to $3=$ brown nuclear staining (equivalent to positive control). Percentage of positive cells was calculated as (numerator=product of antigen-positive cell count and intensity, denominator $=$ total cell count). All fields were evaluated if sample size precluded 16 fields.

\section{Statistical analysis}

Analyses were conducted with IBM SPSS Statistics V.20 software (IBM Corporation, Armonk, New York, USA). MMR protein to lesion severity relationship was tested by Spearman's rank correlation after ordinal grading of lesions: normal, 0 ; LD, 1; HD, 2; well-differentiated OSCC, 3 and poorly differentiated OSCC, 4. Age, sex and lesion site were control variables in the multinomial logistic regression analysis of each MMR protein. Backward stepwise elimination of variables from the saturated multinomial model was performed to achieve a best fit.

\section{RESULTS}

Representative photomicrographs for MMR antigen expression are presented (figure 1). In general, MMR protein expression was highest in normal samples and showed a decreasing trend as lesion severity increased (figure 2). Disease 
Table 1 Patient demographic and clinical information

\begin{tabular}{|c|c|c|c|c|c|c|}
\hline \multirow[b]{3}{*}{ Variable } & \multicolumn{6}{|l|}{ Samples } \\
\hline & \multirow[b]{2}{*}{ Normal mucosa } & \multicolumn{3}{|l|}{ Dysplasia } & \multicolumn{2}{|l|}{ oscc } \\
\hline & & Low risk & High risk & Carcinoma in situ & Well differentiated & Moderately/poorly differentiated \\
\hline \multicolumn{7}{|l|}{ Gender } \\
\hline Male & 52 & 13 & 9 & 7 & 55 & 19 \\
\hline Female & 38 & 24 & 12 & 6 & 31 & 8 \\
\hline \multicolumn{7}{|l|}{ Age at diagnosis } \\
\hline$<45$ years & 57 & 28 & 16 & 11 & 20 & 5 \\
\hline$\geq 45$ years & 33 & 9 & 5 & 2 & 66 & 22 \\
\hline \multicolumn{7}{|l|}{ Biopsy site } \\
\hline Tongue & 9 & 3 & 2 & 1 & 14 & 2 \\
\hline Palate & 9 & 4 & 1 & 0 & 6 & 4 \\
\hline Gingiva & 21 & 9 & 4 & 2 & 15 & 11 \\
\hline Labial mucosa & 12 & 2 & 1 & 0 & 10 & 0 \\
\hline Buccal mucosa & 36 & 14 & 9 & 8 & 29 & 5 \\
\hline Floor of mouth & 2 & 2 & 2 & 2 & 9 & 5 \\
\hline Not available & 1 & 3 & 2 & 0 & 3 & 0 \\
\hline \multicolumn{7}{|l|}{ Smoking history } \\
\hline Positive & 9 & 4 & 6 & 4 & 3 & 4 \\
\hline Negative & 14 & 4 & 0 & 2 & 7 & 5 \\
\hline Unavailable & 67 & 29 & 15 & 7 & 76 & 18 \\
\hline Total & 90 & 37 & 21 & 13 & 86 & 27 \\
\hline
\end{tabular}

OSCC, oral squamous cell carcinoma.

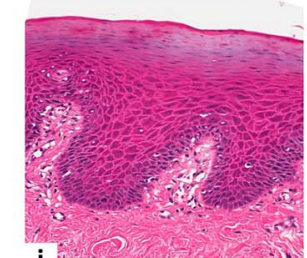

A
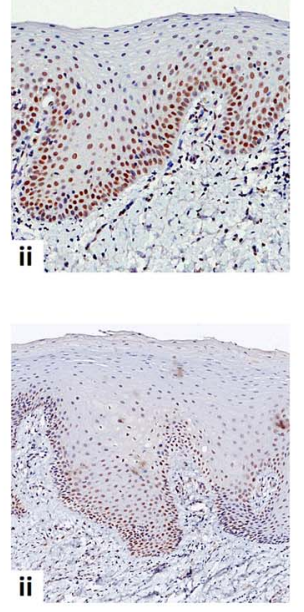

B
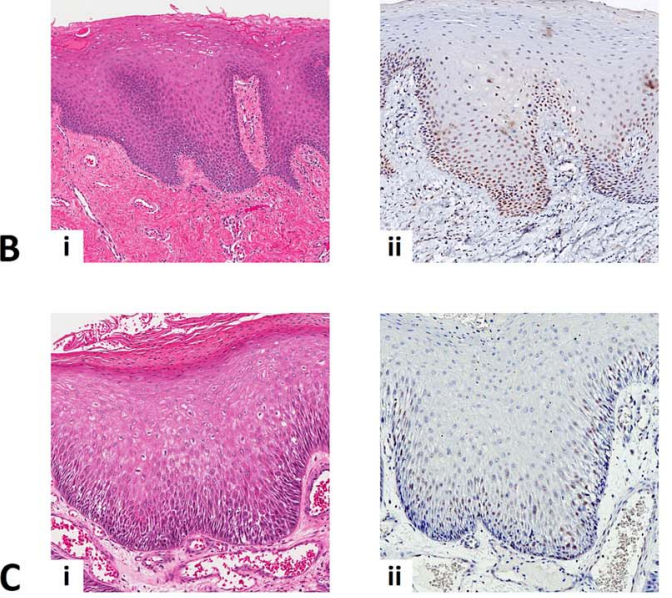
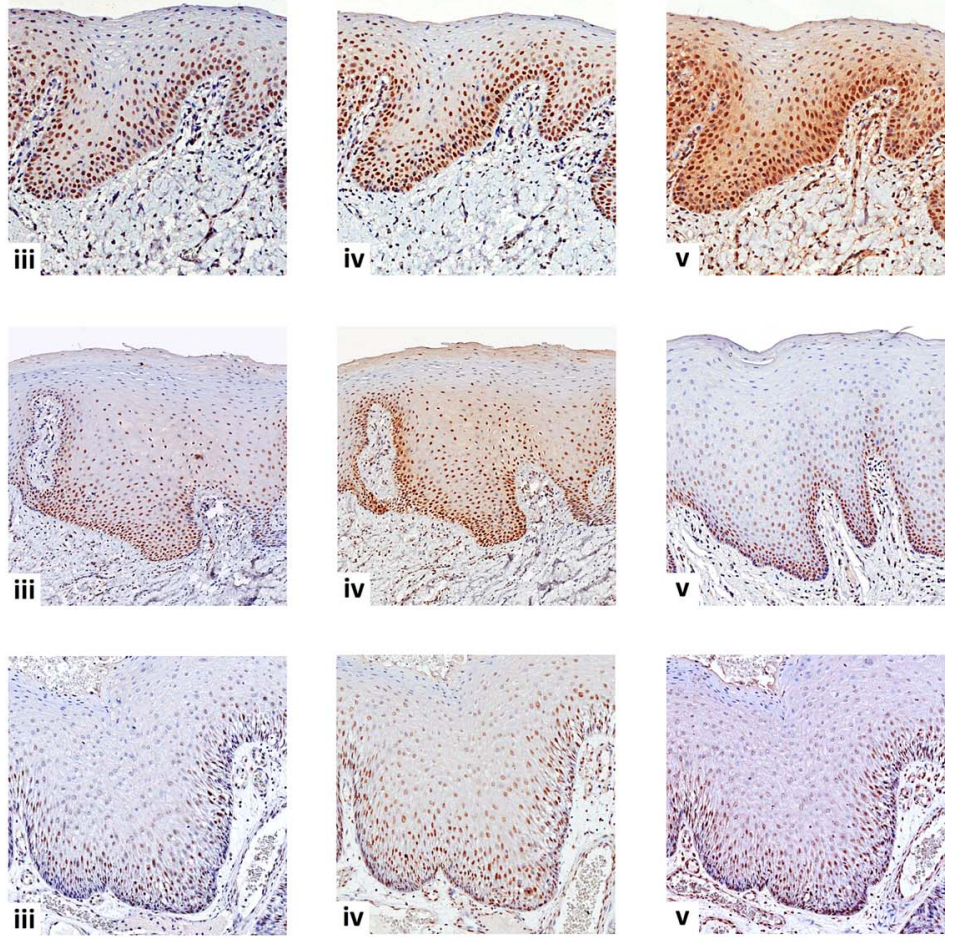

Figure 1 Representative photomicrographs showing (A) normal oral mucosa, (B) low-risk oral epithelial dysplasia, (C) high-risk oral epithelial dysplasia, (D) well-differentiated oral squamous cell carcinoma (OSCC) cell nests and the underlying connective tissue and (E) poorly differentiated OSCC stained with (i) H\&E, (ii) hMLH1, (iii) hPMS2, (iv) hMSH2 and (v) hMSH6. Magnification $\times 20$. 

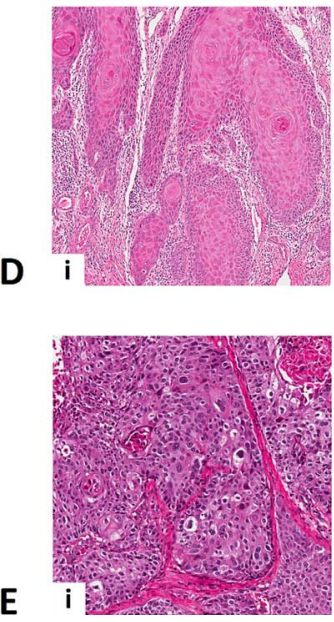
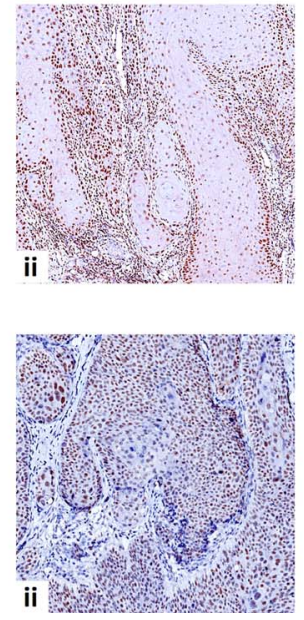
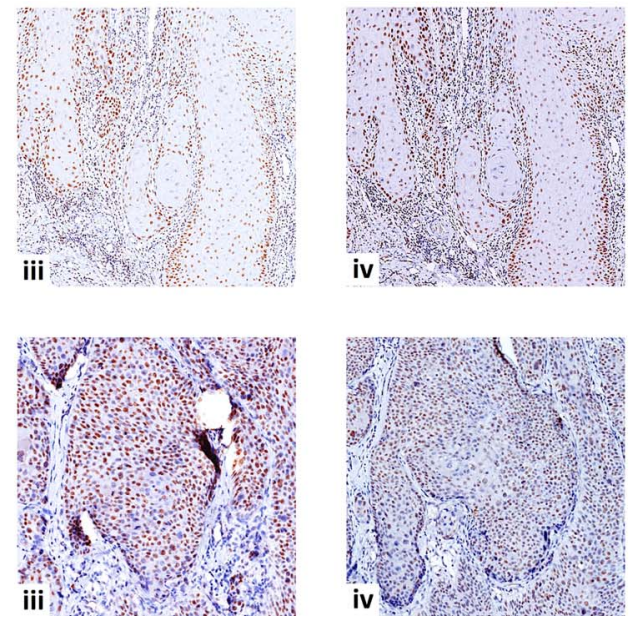
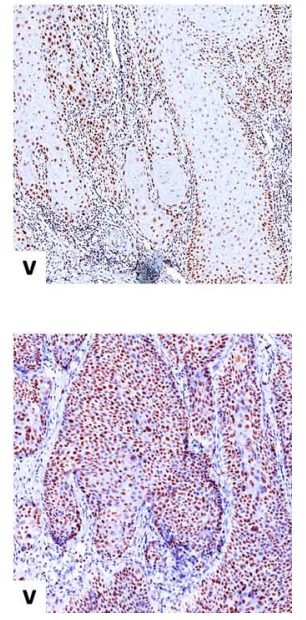

Figure 1 Continued.

severity correlated significantly with expression of: hMLH1 $(\rho=-0.715)$, hPMS2 $(\rho=-0.692)$, hMSH2 $(\rho=-0.728)$ and hMSH6 ( $\rho=-0.702) ;(n=274)$. The majority (nine out of 13) of CIS samples exhibited localised loss of hMSH6 from the stratum basale (figure 3). The complementary obligatory and non-obligatory components of the MutS $\alpha$ and MutL $\alpha$ dimers

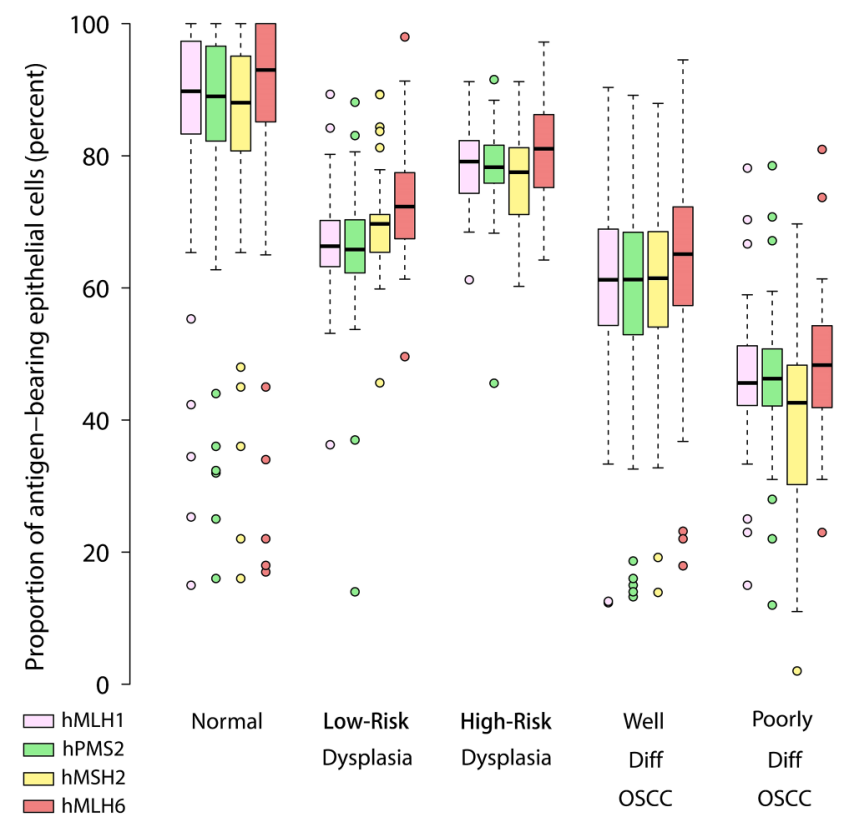

Figure 2 Distribution of immunohistochemical stain intensity for mismatch repair proteins in normal, low-risk dysplasia, high-risk dysplasia, well-differentiated oral squamous cell carcinoma (OSCC) and poorly differentiated OSCC samples. correlated appropriately (hMSH2 and hMSH6, $\rho=0.774$ and hMLH1 and hPMS2, $\rho=0.847$ ).

When analysed individually by multinomial logistic regression, hMLH1, hPMS2 and hMSH2 were significantly associated with disease severity. Expression of hMSH6 was decreased in LD and OSCC but not for HD. In these analyses, MMR heterodimers were significantly decreased in all pathological conditions compared with normal, with an exception of hMSH6 in predicting the risk of HD (table 2). Table 3 presents the final results from backward stepwise elimination of lesion site, hMSH6 and sex from the saturated model. Each per cent increase in expression of hMLH1, decreased the odds of having $\mathrm{LD}(\mathrm{OR}=0.92$, CI 0.88 to 0.97$)$ and well-differentiated OSCCs (OR $=0.92$, CI 0.88 to 0.96 ) by a factor of 0.92 . Similarly, the odds of having poorly differentiated OSCCs were decreased by a factor of $0.86(95 \%$ CI 0.81 to -0.91$)$ with every $1 \%$ increase in expression of hMSH2. One intriguing finding which warrants further investigation was the localised loss of hMSH6 from the stratum basale of CIS specimens.

\section{DISCUSSION}

This study established correlation between loss of MMR protein expression with the severity of OED in OPML and with the degree of differentiation in OSCC. Three important findings emerged. First, a reduction in immunoexpression of the obligatory components of MutS $\alpha$ and MutL $\alpha$ during the progression of pathology from OPML to OSCC was observed. Second, a similar trend was found for expression of the non-obligatory components of MutS $\alpha$ and MutL $\alpha$. Third, there is conspicuous loss of hMSH6 expression from the stratum basale of CIS. These findings are consistent with the breadth of research surrounding MMR protein and specifically address the need for more rigorous techniques to assist with the grading of oral lesions, particularly CIS. 

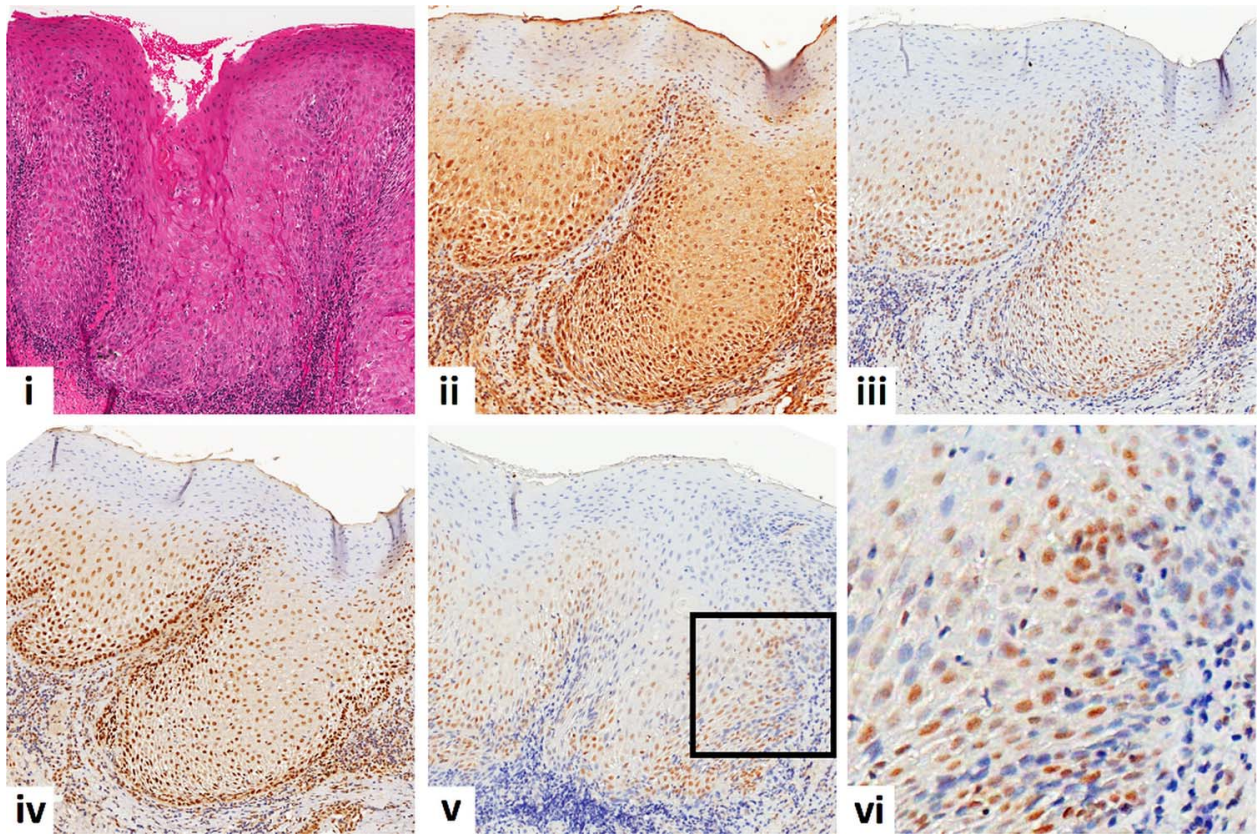

Figure 3 Representative photomicrographs showing carcinoma in situ samples stained with (i) H\&E, (ii) hMLH1, (iii) hPMS2, (iv) hMSH2 and (v) hMSH6. Magnification $\times 20$. Image (vi) represents a high magnification $(\times 40)$ of the insert indicated in image (v) demonstrating clear loss of hMSH6 in the stratum basale.

Expression of hMSH2 and hMLH1 have been investigated previously for OPML and OSCC, ${ }^{13} 14$ however, this study is the first to report simultaneous reduction in expression of both the obligatory and non-obligatory components. These results provide considerable weight to the established role of MMR process impairment during OSCC initiation and progression.

While the obligatory and non-obligatory components of MutS $\alpha$ and MutL $\alpha$ are complementary, they are, nonetheless, discrete gene products that are subject to independent regulation. Here, strong correlations between these obligatory and non-obligatory components have been reported (hMSH2 vs hMSH6, $\rho=0.774$; hMLH1 vs hPMS2, $\rho=0.847$ ). These findings are indicative of a regulatory event that lies upstream of the individual gene expression levels, as opposed to discrete gene mutations or transcriptional modifications. Interestingly, based upon their predictive OR, only three out of the four MMR proteins were retained by the multinomial logistic model; the excluded MMR protein being hMSH6, the nonobligatory MutS $\alpha$ dimer. The low weight of hMSH6 in our statistical analyses can be readily accounted for by its anomalous expression in the majority of CIS cases, whereby hMSH6 expression was lost from the stratum basale of otherwise hMSH6-positive CIS epithelia (figure 3). This novel finding warrants further investigation, since localised loss of hMSH6 has potential to become a diagnostic marker in OED/OPML. Once validated, hMSH6 immunostaining may partially address the current need for additional diagnostic markers to assist with OED grading.

In this study, immunoexpression of MMR proteins in oral lesions was studied individually and as a panel. A stepwise logistic regression was used to (1) study these proteins as a panel and (2) eliminate those without a significant contribution to the model. Our results are suggestive of a diagnostic role for immunoexpression of hMLH1, hPMS2 and hMSH6 in grading of oral lesions individually, and preferably combined as a panel. Immunoexpression of hMSH6 was shown to particularly distinguish CIS samples. Our findings are independent of the statistical model used to prove their significance, and once validated would be of direct relevance to clinical pathologists as applicable biomarkers of oral disease severity to lessen possible diagnostic ambiguity among the more severe grades of OED, CIS and early invasive OSCC.

Table 2 Multinomial logistic regression for single variables with control variables

\begin{tabular}{|c|c|c|c|c|}
\hline & \multicolumn{4}{|c|}{ Parameter estimates OR $(95 \% \mathrm{Cl})$} \\
\hline & \multicolumn{2}{|l|}{ Dysplasia } & \multicolumn{2}{|l|}{ oscc } \\
\hline & Low risk & High risk & Well differentiated & Poorly differentiated \\
\hline hMLH1 & $0.88^{*}(0.84$ to 0.91$)$ & $0.95^{* *}(0.92$ to 0.99$)$ & $0.84^{*}$ (0.81 to 0.88$)$ & $0.74^{*}(0.74$ to 0.83$)$ \\
\hline hPMS2 & $0.89 *(0.86$ to 0.92$)$ & $0.96^{* *}(0.92$ to 0.99$)$ & $0.87^{*}(0.84$ to 0.90$)$ & $0.82^{*}(0.78$ to 0.86$)$ \\
\hline hMSH2 & $0.91 *(0.87$ to 0.94$)$ & $0.95^{* *}(0.92$ to 0.99$)$ & $0.86^{*}(0.83$ to 0.89$)$ & $0.80 *(0.76$ to 0.84$)$ \\
\hline hMSH6 & $0.92 *(0.89$ to 0.95$)$ & $0.96(0.93$ to 1.00$)$ & $0.88 *(0.86$ to 0.91$)$ & $0.84^{*}(0.81$ to 0.88$)$ \\
\hline
\end{tabular}


Table 3 Stepwise backward elimination method of multinomial logistic regression

\begin{tabular}{|c|c|c|c|c|}
\hline \multirow[b]{3}{*}{$\mathrm{N}=$} & \multicolumn{4}{|c|}{ Parameter estimates OR $(95 \% \mathrm{Cl})$} \\
\hline & \multicolumn{2}{|l|}{ Dysplasia } & \multicolumn{2}{|c|}{ Oral squamous cell carcinoma } \\
\hline & $\begin{array}{l}\text { Mild } \\
(n=37)\end{array}$ & $\begin{array}{l}\text { Moderate/severe } \\
(n=34)\end{array}$ & $\begin{array}{l}\text { Well differentiated } \\
(n=86)\end{array}$ & $\begin{array}{l}\text { Poorly differentiated } \\
(n=27)\end{array}$ \\
\hline \multicolumn{5}{|c|}{ Variables in the model } \\
\hline hMLH1 & $0.92^{* *}(0.88$ to 0.97$)$ & 0.97 (0.92 to 1.03$)$ & $0.92 *$ (0.88 to 0.96$)$ & $0.92^{* *}(0.85$ to 1.00$)$ \\
\hline hPMS2 & $0.94^{* *}(0.91$ to 0.98$)$ & 0.98 (0.94 to 1.03$)$ & $0.94^{*}(0.90$ to 0.97$)$ & $0.89^{*}(0.84$ to 0.95$)$ \\
\hline hMSH2 & $0.96(0.92$ to 1.00$)$ & 0.97 (0.93 to 1.01 ) & $0.93^{* *}(0.89$ to 0.97$)$ & $0.86^{*}(0.81$ to 0.91$)$ \\
\hline \multicolumn{5}{|c|}{ Control variables } \\
\hline Age & 1.02 (0.98 to 1.05$)$ & $1.04^{* *}(1.00$ to 1.07$)$ & $1.06^{* *}$ (1.02 to 1.09$)$ & $1.07^{* *}(1.02$ to 1.13$)$ \\
\hline
\end{tabular}

The reference group is normal; ${ }^{*} p<0.0001,{ }^{*} p<0.05$. The general formula by which the model was designed is as follows: $\operatorname{Ln}(p(D x) / p($ Normal $))=b+\sum\left(\beta_{x i} \times x i\right)$ in which $\mathrm{p}=$ probability, Dx=Diagnosis of sample (mild dysplasia, moderate/severe dysplasia, well-differentiated OSCC and poorly differentiated OSCC), $b=$ =interface, $\beta=$ coefficient for each independent variable, $\mathrm{x}=$ =independent variable and $\mathrm{i}=$ score for which model is calculated. Following equations were used for data shown in the table:

$$
\begin{aligned}
& \operatorname{Ln}\left(\frac{\text { probability (mild dysplasia) }}{\text { probability (normal) }}\right)=0.54+0.022 \text { age }-0.075 \text { hMLH1 }-0.056 \text { hPMS2 }-0.034 \text { hMSH2 } \\
& \operatorname{Ln}\left(\frac{\text { probability (moderate to severe dysplasia) }}{\text { probability }(\text { normal })}\right)=3.164+0.037 \text { age }-0.028 \text { hMLH1 }-0.016 \text { hPMS } 2-0.032 \text { hMSH2 } \\
& \operatorname{Ln}\left(\frac{\text { probability (well differentiated OSCC) }}{\text { probability }(\text { normal })}\right)=13.808+0.050 \text { age }-0.088 \text { hMLH1 }-0.070 \text { hPMS2 }-0.0569 \text { hMSH2 } \\
& \operatorname{Ln}\left(\frac{\text { probability ( poorly differentiated OSCC) }}{\text { probability }(\text { normal })}\right)=16.659+0.079 \text { age }-0.088 \text { hMLH1 }-0.102 \text { hPMS2 }-0.148 \text { hMSH2 }
\end{aligned}
$$

OSCC, oral squamous cell carcinoma.

\section{Take home message}

Although immunoexpression of all MMR proteins decreased with increasing lesion severity, isolated loss of expression of hMSH6 in the basal cell layer of carcinoma in situ lesions could potentially be a useful diagnostic biomarker for these lesions.

\section{Acknowledgements The authors thank the Australian Dental Research} Foundation for funding this work.

Contributors MJ: immunostaining; scoring; statistical analysis and preparing the first draft. AJD: revision of the first draft; help in designing the experiment and help in presenting the data. CSF: supervision, confirming diagnosis of all H\&E slides, checking of IHC scoring, help in designing the experiment, final proof reading and editing of the manuscript.

Funding Australian Dental Research Foundation.

\section{Competing interests None.}

Ethics approval The Ethics Committees of the University of Queensland (2007001478) and the Royal Brisbane Hospital (HREC/10/QRBW336).

Provenance and peer review Not commissioned; externally peer reviewed.

\section{REFERENCES}

1 Benachenhou N, Guiral S, Gorska-Flipot I, et al. High resolution deletion mapping reveals frequent allelic losses at the DNA mismatch repair loci hMLH1 and hMSH3 in non-small cell lung cancer. Int J Cancer 1998;77:173-80.

2 Fishel R, Wilson T. MutS homologs in mammalian cells. Curr Opin Genet Dev 1997;7:105-13.

3 Modrich P, Lahue R. Mismatch repair in replication fidelity, genetic recombination, and cancer biology. Annu Rev Biochem 1996;65:101-33.
4 Dost F, Lê Cao K, Ford PJ, et al. Malignant transformation of oral epithelial dysplasia: a real-world evaluation of histopathologic grading. Oral Surg Oral Med Oral Pathol Oral Radiol 2014;117:343-52.

5 Kujan O, Oliver RJ, Khattab A, et al. Evaluation of a new binary system of grading oral epithelial dysplasia for prediction of malignant transformation. Oral Oncol 2006;42:987-93.

6 Koutsimpelas D, Pongsapich W, Heinrich U, et al. Promoter methylation of MGMT, MLH1 and RASSF1A tumor suppressor genes in head and neck squamous cell carcinoma: pharmacological genome demethylation reduces proliferation of head and neck squamous carcinoma cells. Oncol Rep 2012; 27:1135-41.

7 Sengupta S, Chakrabarti S, Roy A, et al. Inactivation of human MutL homolog 1 and MutS homolog 2 genes in head and neck squamous cell carcinoma tumors and leukoplakia samples by promoter hypermethylation and its relation with microsatellite instability phenotype. Cancer 2007;109:703-12.

8 Lo Muzio L, Nocini P, Mignogna MD, et al. Immunocytochemical detection of hMSH2 and hMLH1 expression in oral SCC. Anticancer Res 1999; 19:933-40.

9 Tawfik HM, El-MaqsoudNMRA, Hak BHAA, et al. Head and neck squamous cell carcinoma: mismatch repair immunohistochemistry and promoter hypermethylation of hMLH1 gene. Am J Otolaryngol 2011;32:528-36.

10 Caldeira PC, Aguiar MCF, Mesquita RA, et al. Oral leukoplakias with different degrees of dysplasia: Comparative study of hMLH1, p53, and AgNOR. J Oral Pathol Med 2011;40:305-11.

11 Caldeira PC, Abreu MHNG, Batista AC, et al. hMLH1 immunoexpression is related to the degree of epithelial dysplasia in oral leukoplakia. J Oral Pathol Med 2011;40:153-59.

12 Barnes L, Eveson JW, Reichart P, et al. World Health Organization Classification of Tumours. Pathology and Genetics of Head and Neck Tumours. 9th edn. Lyon: IARC Press, 2005.

13 Czerninski R, Krichevsky S, Ashhab Y, et al. Promoter hypermethylation of mismatch repair genes, hMLH1 and hMSH2 in oral squamous cell carcinoma. Oral Dis 2009;15:206-13.

14 Jessri M, Dalley AJ and Farah CS. MutS $\alpha$ and MutL $\alpha$ immunoexpression analysis in diagnostic grading of oral epithelial dysplasia and squamous cell carcinoma. Oral Surgery, Oral Medicine, Oral Pathology and Oral Radiology Published Online First: 25 Sep 2014. 